\title{
A MODIFIED LEACH ALGORITHM USING HARD AND SOFT THRESHOLD IN WIRELESS SENSOR NETWORK : A REVIEW
}

\author{
Gurpreet Kaur $^{(1)}$, Vishal Arora ${ }^{(2)}$ \\ ${ }^{(1)}$ Research Scholar, Department of Computer Science Engineering, SBSSTC, Ferozepur \\ sidhugurpreet22@gmail.com \\ (2) Assistant Professor, Department of Computer Science Engineering, SBSSTC, Ferozepur \\ vishal.fzr@gmail.com
}

\begin{abstract}
Wireless sensor networks have become increasingly popular due to their wide range of application. Clustering sensor nodes organizing them hierarchically have proven to be an effective method to provide better data aggregation and scalability for the sensor network while conserving limited energy. Minimizing the energy consumption of a wireless sensor network application is crucial for effective realization of the intended application in terms of cost, lifetime, and functionality. However, the minimizing task is hardly possible as no overall energy cost function is available for optimization. In this paper, we have proposed a modified alogirthm of leach where hard and soft threshold values will be applied for improving the overall throughput and network lifetime.
\end{abstract}

\section{Keywords}

Leach, Wireless, Sensor, Networks, Protocol, Cluster Head, Threshold, WSN.

\section{Council for Innovative Research}

Peer Review Research Publishing System

Journal: INTERNATIONAL JOURNAL OF COMPUTERS \& TECHNOLOGY

Vol. 14 , No. 10

www.ijctonline.com, editorijctonline@gmail.com 


\section{INTRODUCTION}

Due to recent technological advances, the manufacturing of small and low cost sensors became technically and economically feasible. The sensing electronics measure ambient conditions related to the environment surrounding the sensor and transforms them into an electric signal. Processing such a signal reveals some properties about objects located and/or events happening in the vicinity of the sensor. A large number of these disposable sensors can be networked in many applications that require unattended operations. A Wireless Sensor Network (WSN) contain hundreds or thousands of these sensor nodes. These sensors have the ability to communicate either among each other or directly to an external base-station (BS). Wireless Sensor Network (WSN) is a type of wirelessAd-Hoc network in which large numbers of sensor nodesare deployed in the application field [1]. Sensor nodes are inexpensive and low power devices. Each node consists of four main units: Sensing Unit, ProcessingUnit, Communicating Unit and Power Unit. In sensingunit one or more sensors are placed to sense differentenvironment parameters like sound, temperature,vibration, pressure, motion and etc., based on applicationrequirement. Sensed data's (analog or digital) areprocessed and aggregated in the processing unit.

The need for energy-efficient infrastructures for sensor networks is becoming increasingly important. Wireless sensor networks are networks consisting of many sensor nodes that communicate over a wireless media. A sensor node is equipped with a sensor module, a processor, a radio module and a battery. Since the battery limits the lifetime of the sensor nodes it also limits the lifetime of the sensor network, thus energy efficiency is a major issue for sensor networks. An important goal in many sensor networks is to monitor an area as long time as possible. Hence, it is important to distribute energy consumption evenly across the network. When the energy consumption is evenly distributed, the major part of the sensor nodes will stay alive approximately equally long time. This enables continued information gathering throughout the whole network area during the lifetime of the network. The most power-consuming activity of a sensor node is typically radio communication [2]. Hence, radio communication must be kept to an absolute minimum. This means that the amount of network traffic should be minimized. In order to reduce the amount of traffic in the network, we build clusters of sensor nodes as proposed in e.g. [3,5]. Some sensor nodes become cluster heads and collect all traffic from their respective cluster. The cluster head aggregates the collected data and then sends it to its base station. When using clustering, the workload on the cluster head is thus larger than for non-cluster heads. The cluster heads should therefore be changed several times during the lifetime of the sensor network in order to distribute the extra workload and energy consumption evenly.

Networking unattended sensor nodes may have profound e®ect on the efficiency of many military and civil applications such as target field imaging, intrusion detection, weather monitoring, security and tactical surveillance, distributed computing, detecting ambient conditions such as temperature, movement, sound, light, or the presence of certain objects, inventory control, and disaster management. Deployment of a sensor network in these applications can be in random fashion (e.g., dropped from an airplane) or can be planted manually (e.g., fire alarm sensors in a facility). For example, in a disaster management application, a large number of sensors can be dropped from a helicopter. Networking these sensors can assist rescue operations by locating survivors, identifying risky areas, and making the rescue team more aware of the overall situation in the disaster area. In the past few years, an intensive research that addresses the potential of collaboration among sensors in data gathering and processing and in the coordination and management of the sensing activity were conducted. However, sensor nodes are constrained in energy supply and bandwidth. Thus, innovative techniques that eliminate energy inefficiencies that would shorten the lifetime of the network are highly required. Such constraints combined with a typical deployment of large number of sensor nodes pose many challenges to the design and management of WSNs and necessitiate energy-awareness at all layers of the networking protocol stack. For example, at the network layer, it is highly desirable to find methods for energy-efficient route discovery and relaying of data from the sensor nodes to the BS so that the lifetime of the network is maximized. Routing in WSNs is very challenging due to the inherent characteristics that distinguish these networks from other wireless networks like mobile ad hoc networks or cellular networks. First, due to the relatively large number of sensor nodes, it is not possible to build a global addressing scheme for the deployment of a large number of sensor nodes as the overhead of ID maintenance is high. Thus, traditional IP-based protocols may not be applied to WSNs. Furthermore, sensor nodes that are deployed in an ad hoc manner.

Sensor networks represent a significant improve-ment over traditional sensors, which are deployed in the following two ways [3]:

- Sensors can be positioned far from the actual phenomenon, i.e., something known by sense perception. In this approach, large sensors that use some complex techniques to distinguish the targets from environmental noise are required.

- Several sensors that perform only sensing can be deployed. The positions of the sensors and communications topology are carefully engineered. They transmit time series of the sensed phenomenon to the central nodes where computations are performed and data are fused.

\section{SENSOR NETWORKS APPLICATIONS}

Sensor networks may consist of many different types of sensors such as seismic, low sampling rate magnetic, thermal, visual, infrared, acoustic and radar, which are able to monitor a wide variety of ambient conditions that include the following [23]:

- temperature,

- humidity, 
- vehicular movement,

- lightning condition,

- pressure,

- soil makeup,

- noise levels,

- the presence or absence of certain kinds of objects,

- mechanical stress levels on attached objects, and

- the current characteristics such as speed, direction, and size of an object.

Sensor nodes can be used for continuous sensing, event detection, event ID, location sensing, and local control of actuators. The concept of micro-sensing and wireless connection of these nodes promise many new application areas. We categorize the applications into military, environment, health, home and other commercial areas. It is possible to expand this classification with more categories such as space exploration, chemical processing and disaster relief.

\section{ROUTING CHALLENGES AND DESIGN ISSUES IN WSNS}

Despite the innumerable applications of WSNs, these networks have several restrictions, e.g., limited energy supply, limited computing power, and limited bandwidth of the wireless links connecting sensor nodes. One of the main design goals of WSNs is to carry out data communication while trying to prolong the lifetime of the network and prevent connectivity degradation by employing aggressive energy management techniques. The design of routing protocols in WSNs is in ${ }^{\circ}$ enced by many challenging factors. These factors must be overcome before etcient communication can be achieved in WSNs. In the following, we summarize some of the routing challenges and design issues that a®ect routing process in WSNs.

Node deployment: Node deployment in WSNs is application dependent and affects the performance of the routing protocol. The deployment can be either deterministic or randomized. In deterministic deployment, the sensors are manually placed and data is routed through pre-determined paths.

Energy consumption without losing accuracy: sensor nodes can use up their limited supply of energy performing computations and transmitting information in a wireless environment. As such, energyconserving forms of communication and computation are essential. Sensor node lifetime shows a strong dependence on the battery lifetime [1].

Data Reporting Model: Data sensing and reporting in WSNs is dependent on the application and the time criticality of the data reporting. Data reporting can be categorized as either time-driven (continuous), event-driven, query-driven, and hybrid [13].

Node/Link Heterogeneity: In many studies, all sensor nodes were assumed to be homogeneous, i.e., having equal capacity in terms of computation, communication, and power. However, depending on the application a sensor node can have different role or capability. The existence of heterogeneous set of sensors raises many technical issues related to data routing.

Fault Tolerance: Some sensor nodes may fail or be blocked due to lack of power, physical damage, or environmental interference. The failure of sensor nodes should not affect the overall task of the sensor network. If many nodes fail, MAC and routing protocols must accommodate formation of new links and routes to the data collection base stations.

Scalability: The number of sensor nodes deployed in the sensing area may be in the order of hundreds or thousands, or more. Any routing scheme must be able to work with this huge number of sensor nodes

Network Dynamics: Most of the network architectures assume that sensor nodes are stationary. However, mobility of both BS's or sensor nodes is sometimes necessary in many applications [19].

\section{RELATED WORK}

Jiwa Abdullah [1] discusses that the deployment of Wireless Sensor Network(WSN) must be done with some kind of advanced techniques so that it can mitigate the energy constraints. He modified the LEACH algorithm, the improvement was done in the Cluster Head selection process. The selection process takes into account the residual energy of each node and used its probability outcome for the determination of the threshold value for next round.

Rong Ding [2] presents a novel cluster-head selection algorithm. Instead of changing the threshold to 0 directly, he proposed an algorithm which adjusts the threshold of each node gradually according to the roles they have played in the last round, so more nodes could have the opportunity to be $\mathrm{CHs}$.

R.A. Roseline [3] proposed a hybrid routing algorithm called LCTS(Local Clustering and Threshold Sensitive), that takes the advantages of LEACH and TEEN and eliminates its drawbacks. LCTS makes use of symmetric propagation channels where Cluster Head $(\mathrm{CH})$ advertisement heard with the largest signal strength is the cluster-head to whom minimum amount of transmitted energy is needed for communication. The Base Station (BS) selects the Cluster Heads based on location and then the clusters are formed by the Cluster Heads. The nodes communicate with the cluster head in TDMA 
and send their energy level even if the threshold is not reached and this ensures that the nodes are alive. The sensed data is compressed at the $\mathrm{CH}$ and sent to the Base Station.

Zahra Beiranvand [4] proposed, an energy efficient routing algorithm which saves a significant portion of innernetwork communications energy. To do this, the proposed routing algorithm selects sensor nodes with higher residual energy, more neighbors, and lower distance from the Base Station (BS) as Cluster Head (CH) nodes. Then, it manages sensor nodes appropriately and constructs clusters such a way to maximize WSN lifetime and minimize average energy dissipation per each sensor node.

Deepali [5] proposes a new routing protocol based on sink mobility i.e. improved EESSC (IEESSC). This protocol is simulated and compared with EESSC and LEACH based on parameters stability period, network lifetime and middle node death (MND).

D. Mahmood [6] modified one of the most prominent wireless sensor network's routing protocol "LEACH" as modified LEACH (MODLEACH) by introducing efficient cluster head replacement scheme and dual transmitting power levels. Our modified LEACH, in comparison with LEACH out performs it using metrics of cluster head formation, through put and network life. Afterwards, hard and soft thresholds are implemented on modified LEACH (MODLEACH) that boast the performance even more.

Bao Zhenshah [7] proposed hierarchy tree routing based on LEACH (HT-LEACH). The algorithm defines three phrases in each round: cluster formation, hierarchy tree routing formation and steady-state. In the second phrase, hierarchy tree routing is built in cluster heads.

Zhong Liu [8] improves the cluster-head selection mechanism, it considers the residual energy of nodes, distance to sink node and range of clusters. It optimizes cluster-head selection by Chaos-PSO. Then the other nodes decide whether join the cluster or not by comparing the signal strength of advertisement message with a predefined threshold, and the range of cluster is restricted.

Liu Zun [9] aims to the disadvantage of the short lifetime due to the uneven distribution of the cluster head, and single-hop path communication style in the LEACH protocol. By analyzing the classic clumps and routing protocol LEACH, and changing the way of producing cluster head and the communication way between the base station ,the modified algorithm reduces the setup time and the cluster head communication distance and balances the node energy consumption .

Julien Bernard [10], The study given by the author exhibit two kinds of performance sensitivity regarding simulations: the first one concerns the communication model itself and the second one is generated by the parameters of the communication model. He provide a generalized model that covers very different situations and we state the routing problem as a linear programming problem in order to measure the absolute efficiency of the algorithms with different models and parameter values.

\section{PROPOSED WORK}

In the proposed paper, we have modified the Leach algorithm by using the Soft and Hard threshold values. The amplification energy used for transmission will be adjusted dynamically. The transmission amplification energy is different for Intra-Cluster transmission, inter-cluster transmission and Cluster head to base station transmission. We will be using different levels of amplification energy along with soft threshold value and high threshold value. The proposed routing algorithm uses shortest energy cost path that maintained the energy balance for entire network. Node that has minimum battery power will drain out their battery power quickly and would be the first one to die. When network is setup each node can broadcast their residual energy information. All the nodes in network know the residual energy of neighbouring nodes. All the nodes will have different levels of amplification energy depending upon whether it is in the near zone or the far zone. The objective is to find out best energy efficient algorithm that will lead to the maximization of system lifetime.

\section{CONCLUSION}

In this paper, we presented a new approach for minimizing the total energy consumption of wireless sensor network applications based on the soft and hard threshold for improving the overall network lifetime. We will implementing the above said algorithm in matlab environment

\section{REFERENCES}

[1] Jiwa Abdullah, Maximizing the Network Lifetime of Clustered-based WS N Using Probability of Residual Energy, 2014, IEEE, IEEE International Conference on Control System, Computing and Engineering, November 2014.

[2] Rong Ding, Bing Yang, Lei Yang, Jiawei Wang, Soft Threshold Based Cluster-head Selection Algorithm for Wireless Sensor Networks, 2009 Third International Conference on Sensor Technologies and Applications, IEEE.

[3] R.A.Roseline, P.Sumathe, Local Clustering and Threshold Sensitive routing algorithm for Wireless Sensor Networks, IEEE.

[4] Zahra Beiranvand, Ahmad Patooghy, Mahdi Fazeli, I-LEACH: An Efficient Routing Algorithm to Improve Performance \& to Reduce Energy Consumption in Wireless Sensor Networks, IEEE, 2013 5th Conference on Information and Knowledge Technology (IKT). 
[5] Deepali, Padmavati, Improved Energy Efficiency semi static routing algorithm using sink mobility for WSNs, IEEE, Proceedings of 2014 RAECS UIET Panjab University Chandigarh.

[6] D. Mahmood, N.Javaid, S.Mahmood, S. Qureshi, A.M.Memon, T. Zaman, MODLEACH: A Variant of LEACH for WSNs, IEEE, 2013 Eighth International Conference on Broadband, Wireless Computing, Communication and Applications.

[7] Bao Zhenshan, Xue Bo, Zhang Wenbo, HT-LEACH: An Improved Energy Efficient Algorithm Based on LEACH, IEEE, 2013, International Conference on Mechatronic Sciences, Electric Engineering and Computer (MEC).

[8] Zhong Liu, ZhiKun Liu, and Lin Wen, A Modified LEACH Protocol for Wireless Sensor Networks, IEEE, Fourth International Workshop on Advanced Computational Intelligence Wuhan, 2011.

[9] Liu Jun, Qi Hua, Li Yan, A Modified LEACH algorithm In Wireless Sensor Network Based On NS2, IEEE, 2012 International Conference on Computer Science and Information Processing (CSIP).

[10] Julien Bernard, Violeta Felea, Performance Sensitivity of Routing Algorithms with Various Models of Wireless Sensor Networks, 2013, IEEE. 